\title{
Electric field generated by transition radiation of a short bunch
}

\author{
G. Stupakov \\ SLAC National Accelerator Laboratory, Menlo Park, CA 94025
}

April 14, 2013

\section{INTRODUCTION}

A frequently used method for generation of terahertz wavelengths is based on transition radiation. A relativistic beam impinges a metal foil at 45 degrees

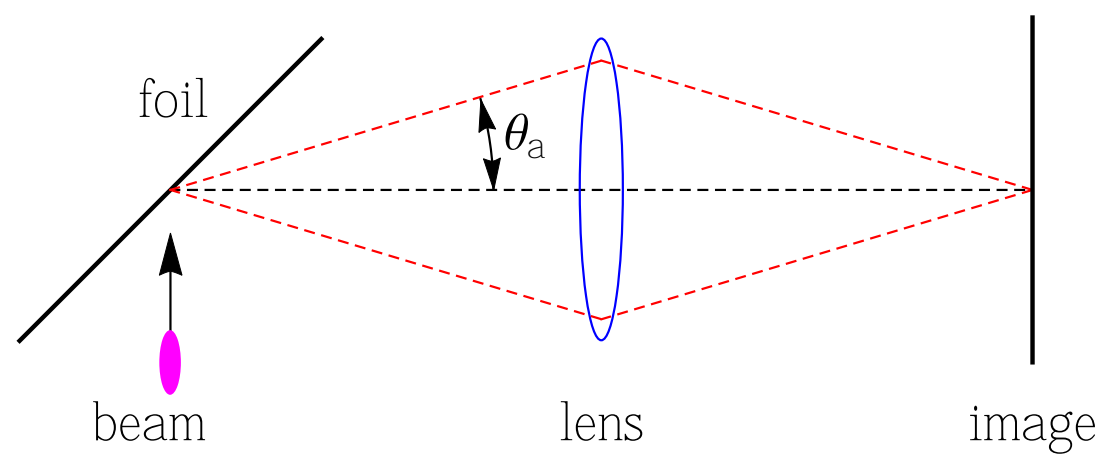

FIG. 1. Transition radiation of a relativistic beam from a metal foil is focused onto the image plane.

and the radiation is collected and focused onto an image plane at 90 degrees relative to the beam orbit. While there are computer codes available to calculate the electric field at the focus $[1,2]$, it is desirable to have a simple theory that would allow a quick estimate of the fields and provide scaling relations between the beam parameters and those of the radiation. The goal of this paper is to develop such a theory. It follows the original calculations done by 
Lebedev [3] and Loos [4] and extends those from the frequency to the time domain.

While in reality the transport line for the $\mathrm{THz}$ radiation may have several focusing elements, to simplify analysis, we consider the setup shown in Fig. 1 with a single lens that provides the focusing. The numerical aperture of the lens $\theta_{a}$ is assumed to be much larger than the inverse gamma factor, $\theta_{a} \gg 1 / \gamma$.

\section{TRANSITION RADIATION FROM METAL SURFACE}

To calculate the radiation field we use the standard approach based on the vectorial diffraction theory [5-7]. To avoid unnecessary geometric complications, we will actually analyze the case of backward transition radiation shown in Fig. 2, which is equivalent to the real setup of Fig. 1. We assume that the
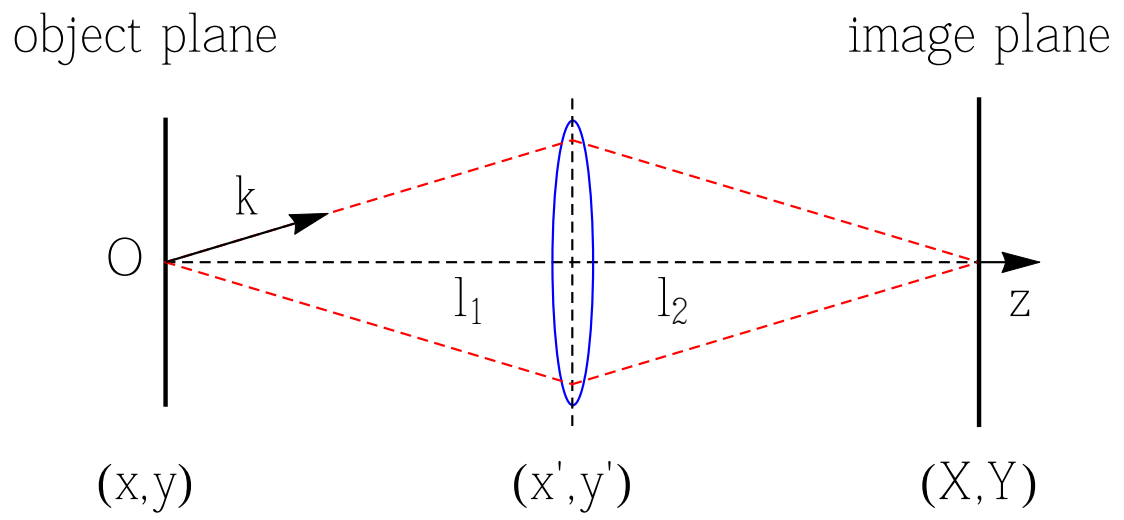

FIG. 2. Equivalent setup. The origin of the coordinate system is located at point $\mathrm{O}$ in the object plane. Shown are notations for the transverse coordinates in each plane.

transverse size of the foil is large enough and carry out integrations in the foil plane from minus to plus infinity. The lens is assumed to be infinitely thin. In 
what follows, we neglect the transverse size of the beam (but take into account its finite length).

All fields are assumed to have the time dependence $\propto e^{-i \omega t}$. The electric field $\boldsymbol{E}_{\mathrm{rad}}\left(\boldsymbol{r}^{\prime}\right)$ in the plane of the lens at distance $l_{1}$ from the foil is given by the following equation,

$$
\boldsymbol{E}_{\mathrm{rad}}\left(\boldsymbol{r}^{\prime}\right)=\frac{e^{i k \mathcal{R}}}{\mathcal{R}} \frac{i}{2 \pi} \boldsymbol{k} \times \int_{\mathrm{pl}} e^{-i \boldsymbol{k} \cdot \boldsymbol{r}} \hat{\boldsymbol{z}} \times \mathcal{E}(\boldsymbol{r}) d^{2} r,
$$

where $\boldsymbol{r}=(x, y)$ is the two-dimensional vector in the plane of the metal (see Fig. 2), $\boldsymbol{r}^{\prime}=\left(x^{\prime}, y^{\prime}\right)$ is the vector in the plane of the lens, $k=|\boldsymbol{k}|=\omega / c$, vector $\boldsymbol{k}$ is directed from the origin $\mathrm{O}$ to point $\boldsymbol{r}$ in the lens plane, $\hat{\boldsymbol{z}}$ is the unit vector perpendicular to the surface of the metal, $\mathcal{E}(\boldsymbol{r})$ is the electric field of the incident electron on the metal surface, and the sign $\int_{\mathrm{pl}}$ indicates that the integration goes over the whole object plane. The quantity $\mathcal{R}$ is the distance between the origin of the coordinate system $(0,0)$ on the metal and the point $\left(x^{\prime}, y^{\prime}\right)$ on the lens, and is approximately equal to

$$
\mathcal{R}=\sqrt{l_{1}^{2}+r^{\prime 2}} \approx l_{1}+\frac{r^{\prime 2}}{2 l_{1}}
$$

where we assumed that $l_{1}$ is much greater than the transverse size of the lens. In the small-angle approximation Eq. (1) can be simplified,

$$
\boldsymbol{E}_{\mathrm{rad}}\left(\boldsymbol{r}^{\prime}\right)=-e^{i k l_{1}+i k r^{\prime 2} / 2 l_{1}} \frac{i k}{2 \pi l_{1}} \int_{\mathrm{pl}} e^{-i k \boldsymbol{r}^{\prime} \cdot \boldsymbol{r} / l_{1}} \mathcal{E}(\boldsymbol{r}) d^{2} r
$$

Considering (3) as a 2D Fourier integral we can use the inverse Fourier transform to express $\mathcal{E}$ through $\boldsymbol{E}_{\text {rad }}$,

$$
\mathcal{E}=\frac{i k}{2 \pi l_{1}} e^{-i k l_{1}} \int_{\mathrm{pl}} e^{i k \boldsymbol{r}^{\prime} \cdot \boldsymbol{r} / l_{1}} e^{-i k r^{\prime 2} / 2 l_{1}} \boldsymbol{E}_{\mathrm{rad}}\left(\boldsymbol{r}^{\prime}\right) d^{2} r^{\prime} .
$$

We first consider radiation of a single electron in the bunch that hits the metal at the origin of the coordinate system. The reflected field on the surface 
is [5] (the minus sign is due to the requirement that the total field on the surface is zero)

$$
\mathcal{E}=-\frac{k e}{c \pi \gamma} K_{1}\left(\frac{k r}{\gamma}\right) \boldsymbol{\nu}
$$

with $\boldsymbol{\nu}=\boldsymbol{r} / r$. It is clear that $\boldsymbol{E}_{\text {rad }}$ is directed along $\boldsymbol{r}^{\prime}$, that is $\boldsymbol{E}_{\text {rad }}\left(\boldsymbol{r}^{\prime}\right)=$ $E_{\mathrm{rad}}\left(r^{\prime}\right) \boldsymbol{r}^{\prime} / r^{\prime}$. For $E_{\mathrm{rad}}$ we obtain

$$
\begin{aligned}
E_{\mathrm{rad}} & =e^{i k l_{1}+i k r^{\prime 2} / 2 l_{1}} \frac{i}{2 \pi l_{1}} \frac{k^{2} e}{c \pi \gamma} \int_{\mathrm{pl}} d^{2} r K_{1}\left(\frac{k r}{\gamma}\right) e^{-i k \boldsymbol{r}^{\prime} \cdot \boldsymbol{r} / l_{1}} \boldsymbol{\nu} \cdot \frac{\boldsymbol{r}^{\prime}}{r^{\prime}} \\
& =e^{i k l_{1}+i k r^{\prime 2} / 2 l_{1}} \frac{i}{2 \pi l_{1}} \frac{k^{2} e}{c \pi \gamma} \int_{0}^{\infty} r d r \int_{0}^{2 \pi} d \phi K_{1}\left(\frac{k r}{\gamma}\right) e^{-i k r^{\prime} r \cos \phi / l_{1}} \cos \phi
\end{aligned}
$$

The integral over $\phi$ can be taken using

$$
\int_{0}^{2 \pi} e^{-i \alpha \cos \phi} \cos \phi d \phi=-2 \pi i J_{1}(\alpha)
$$

with the result

$$
\begin{aligned}
E_{\mathrm{rad}}\left(r^{\prime}\right) & =e^{i k l_{1}+i k r^{\prime 2} / 2 l_{1}} \frac{k^{2} e}{c \pi \gamma l_{1}} \int_{0}^{\infty} K_{1}\left(\frac{k r}{\gamma}\right) J_{1}\left(\frac{k r r^{\prime}}{l_{1}}\right) r d r \\
& =e^{i k l_{1}+i k r^{\prime 2} / 2 l_{1}} \frac{e}{c \pi l_{1}} \frac{r^{\prime} / l_{1}}{\left(r^{\prime} / l_{1}\right)^{2}+\gamma^{-2}}
\end{aligned}
$$

As we will see in the subsequent analysis, the electric field near the focus is created by the lens regions with $r^{\prime} \sim a$ or, equivalently, $r^{\prime} / l_{1} \sim \theta_{a}$. Since we assume that $\theta_{a} \gg \gamma^{-1}$, it is easy to see that the main contribution to $E_{\mathrm{rad}}\left(r^{\prime}\right)$ in (8) comes from the integration area on the foil where $r \sim 1 / \theta_{a} k$. This justifies using the far-field expression (1) if the following condition is satisfied,

$$
l_{1} \gg \frac{1}{k \theta_{a}^{2}},
$$

which we assume throughout this paper. 


\section{EFFECT OF THE LENS}

The lens has a focal length $f$ and radius $a$ and is located at distance $l_{1}$ from the foil, as shown in Fig. 2. The numerical aperture for the lens $\theta_{a}$ is assumed small, $\theta_{a}=a / l_{1} \ll 1$. The electric field $\boldsymbol{E}(\boldsymbol{R})$ at distance $l_{2}$ from the lens is given by the same equation (3) with the additional phase factor $e^{-i k r^{2} / 2 f}$ due to the refraction in the lens [8],

$$
\boldsymbol{E}(\boldsymbol{R})=-\frac{e^{i k l_{2}}}{l_{2}} \frac{i k}{2 \pi} \int_{\mathrm{lens}} e^{-i k \boldsymbol{R} \cdot \boldsymbol{r}^{\prime} / l_{2}+i k r^{\prime 2} / 2 l_{2}-i k r^{\prime 2} / 2 f} \boldsymbol{E}_{\mathrm{rad}}\left(\boldsymbol{r}^{\prime}\right) d^{2} r^{\prime}
$$

where the integration goes over the lens aperture.

We now substitute (8) into this equation

$$
\begin{aligned}
\boldsymbol{E}(\boldsymbol{R}) & =-\frac{e^{i k l_{2}}}{l_{2}} \frac{i k}{2 \pi} \frac{e}{c \pi l_{1}} \int_{\mathrm{lens}} e^{-i k \boldsymbol{R} \cdot \boldsymbol{r}^{\prime} / l_{2}+i k r^{\prime 2} / 2 l_{2}-i k r^{\prime 2} / 2 f} \\
& \times \frac{\boldsymbol{r}^{\prime} / l_{1}}{\left(r^{\prime} / l_{1}\right)^{2}+\gamma^{-2}} e^{i k l_{1}\left(1+r^{\prime 2} / 2 l_{1}^{2}\right)} d^{2} r^{\prime}
\end{aligned}
$$

and choose the distances $l_{1}$ and $l_{2}$ in such a way that

$$
\frac{1}{l_{1}}+\frac{1}{l_{2}}=\frac{1}{f}
$$

so that the lens images the metal plane onto the observation plane. This choice of $l_{2}$ cancels the quadratic terms in the exponent of the integrand in (11),

$$
\boldsymbol{E}(\boldsymbol{R})=-\frac{e^{i k\left(l_{1}+l_{2}\right)}}{l_{2}} \frac{i k}{2 \pi} \frac{e}{c \pi l_{1}} \int_{\mathrm{lens}} e^{-i k \boldsymbol{R} \cdot \boldsymbol{r}^{\prime} / l_{2}} \frac{\boldsymbol{r}^{\prime} / l_{1}}{\left(r^{\prime} / l_{1}\right)^{2}+\gamma^{-2}} d^{2} r^{\prime}
$$

The integration of (13) is carried out in Appendix A with the result

$$
\boldsymbol{E}(\boldsymbol{R})=e^{i k\left(l_{1}+l_{2}\right)} \frac{e \boldsymbol{\mu}}{\pi c R}\left[1-J_{0}\left(\frac{k R \theta_{a}}{m}\right)\right],
$$

where $m=l_{2} / l_{1}$ is the magnification factor. Note that the field has a zero value at $R=0$, reaches maximum at some finite value of $R$ and decays with $R$ as $1 / R$ at large distances. The field is radially polarized. 
To find the time dependence of the field we need to make the Fourier transform from $\omega$ to $t$ and take into account the charge distribution in the bunch. In what follows we assume a Gaussian bunch with the rms bunch length of $\sigma_{z}$ and the total charge $Q$. The integrand in the Fourier integral is an even function of $\omega$, so in the Fourier transform we can replace $e^{-i \omega t} \rightarrow 2 \cos \omega t$ and integrate over $\omega$ from 0 to infinity. The field should be multiplied by the longitudinal spectrum of the beam $(Q / e) e^{-k^{2} \sigma_{z}^{2} / 2}$. Dropping an unimportant phase factor we then obtain

$$
E(R, t)=\frac{2 Q}{\pi c r} \int_{0}^{\infty} d \omega \cos (\omega t)\left[1-J_{0}\left(k R \theta_{a} / m\right)\right] e^{-k^{2} \sigma_{z}^{2} / 2} .
$$

Using the dimensionless variables $\xi=k R \theta_{a} / m, \tau=\mathrm{cmt} / R \theta_{a}, b=R / \sigma_{z}$, $s=m \sigma_{z} /\left(R \theta_{a}\right)$ we rewrite $(15)$ as

$$
E(R, t)=\frac{2 Q m}{\pi \sigma_{z}^{2} \theta_{a}} \frac{1}{b^{2}} \int_{0}^{\infty} d \xi \cos (\xi \tau)\left[1-J_{0}(\xi)\right] e^{-\xi^{2} s^{2} / 2} .
$$

\section{FIELD NEAR THE FOCUS}

Near the focus the parameter $s=m \sigma_{z} /\left(R \theta_{a}\right) \gg 1$ is large and one can see that the main contribution comes from the region $\xi \ll 1$ where the Bessel function can be expanded, $J_{0}(\xi) \approx 1-\xi^{2} / 4$,

$$
\begin{aligned}
E(R, t) & =\frac{2 Q m}{\pi \sigma_{z}^{2} \theta_{a}} \frac{1}{b^{2}} \frac{1}{4} \int_{0}^{\infty} d \xi \cos (\xi \tau) \xi^{2} e^{-\xi^{2} s^{2} / 2} \\
& =\sqrt{\frac{\pi}{2}} \frac{Q b \theta_{a}^{2}}{2 \pi \sigma_{z}^{2} m^{2}}\left(1-\left(\theta_{a} b \tau / m\right)^{2}\right) e^{-\left(\theta_{a} b \tau / m\right)^{2} / 2} .
\end{aligned}
$$

In the opposite limit, $s \ll 1$, the main contribution comes from the region $\xi \gg 1$ and we can approximate $1-J_{0}(\xi) \approx 1$

$$
\begin{aligned}
E(R, t) & =\frac{2 Q m}{\pi \sigma_{z}^{2} \theta_{a}} \frac{1}{b^{2}} \int_{0}^{\infty} d \xi \cos (\xi \tau) e^{-\xi^{2}\left(m / \theta_{a} b\right)^{2} / 2} \\
& =\sqrt{\frac{\pi}{2}} \frac{2 Q}{\pi \sigma_{z}^{2}} \frac{1}{b} e^{-\left(\theta_{a} b \tau / m\right)^{2} / 2} .
\end{aligned}
$$


The maximum field is reached at $t=0$ (corresponding the the maximal peak current in the beam). To find this field we need to compute

$$
E_{\max }(r, 0)=\frac{2 Q \theta_{a}}{\pi \sigma_{z}^{2} m} G(s)
$$

where

$$
G(s)=s^{2} \int_{0}^{\infty} d \xi\left[1-J_{0}(\xi)\right] e^{-\xi^{2} s^{2} / 2}=\sqrt{\frac{\pi}{2}} s\left[1-e^{-1 / 4 s^{2}} I_{0}\left(\frac{1}{4 s^{2}}\right)\right],
$$

with $I_{0}$ the modified Bessel function of zero order. The function $G$ reaches maximum value $G_{\max }=0.33$ at $s \approx 0.5$, which gives a simple formula for the maximum field

$$
E_{\max }=0.2 \frac{Q \theta_{a}}{\sigma_{z}^{2} m}
$$

Substituting the value $s=0.5$ into (16) and performing numerical integration we find the time distribution of the electric field at the point where it reaches its maximum value. For illustration we show in Fig. 3 the plot of this function versus time for the case of the bunch with $Q=200 \mathrm{pC}$, the rms bunch length $\sigma_{z} / c=20 \mathrm{fs}$ and the numerical aperture $\theta_{a}=0.2 \mathrm{rad}$ (assuming the magnification $m=1$ ). The point $s=0.5$ corresponds to the distance $R=60 \mu \mathrm{m}$ from the center of the focus. The maximal field in this case is $20 \mathrm{MV} / \mathrm{cm}$.

\section{ACKNOWLEDGMENTS}

I would like to thank H. Loos for useful discussions.

This work was supported by the U.S. Department of Energy under Contract No. DE- AC02-76SF00515. 


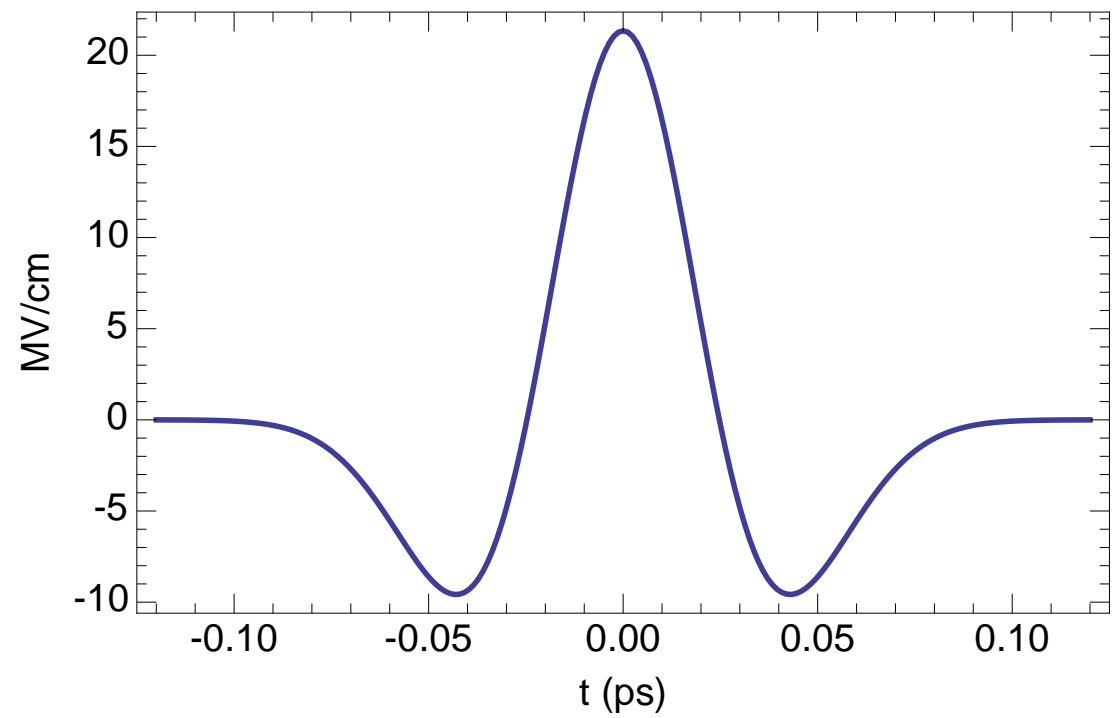

FIG. 3. The dependence of the electric field versus time at the point where it reaches its maximal value.

\section{Appendix A: Calculation of integral (13)}

We rewrite the integral in Eq. (13) as follows

$$
\begin{aligned}
I_{\text {lens }}=\int_{\text {lens }} e^{-i k \boldsymbol{R} \cdot \boldsymbol{r}^{\prime} / l_{2}} \frac{\boldsymbol{r}^{\prime} / l_{1}}{\left(r^{\prime} / l_{1}\right)^{2}+\gamma^{-2}} d^{2} r^{\prime} & =\int_{\mathrm{pl}} e^{-i k \boldsymbol{R} \cdot \boldsymbol{r}^{\prime} / l_{2}} \frac{\boldsymbol{r}^{\prime} / l_{1}}{\left(r^{\prime} / l_{1}\right)^{2}+\gamma^{-2}} d^{2} r^{\prime} \\
& -\int_{\text {out }} e^{-i k \boldsymbol{R} \cdot \boldsymbol{r}^{\prime} / l_{2}} \frac{\boldsymbol{r}^{\prime} / l_{1}}{\left(r^{\prime} / l_{1}\right)^{2}+\gamma^{-2}} d^{2} r^{\prime}
\end{aligned}
$$

where in the first integral on the right hand side the integration goes over the whole plane, and in the second one the integration region lies outside of the lens aperture. The first integral can be easily computed if one notes that 
substituting (8) into (4) one obtains

$$
\mathcal{E}=\frac{i k}{2 \pi l_{1}} \frac{Q}{c \pi l_{1}} \int_{\mathrm{pl}} e^{i k \boldsymbol{r}^{\prime} \cdot \boldsymbol{r} / l_{1}} \frac{\boldsymbol{r}^{\prime} / l_{1}}{\left(r^{\prime} / l_{1}\right)^{2}+\gamma^{-2}} d^{2} r^{\prime},
$$

where $\mathcal{E}$ is given by (5). Hence

$$
\int_{\mathrm{pl}} e^{i k \boldsymbol{r}^{\prime} \cdot \boldsymbol{R} / l_{2}} \frac{\boldsymbol{r}^{\prime} / l_{1}}{\left(r^{\prime} / l_{1}\right)^{2}+\gamma^{-2}} d^{2} r^{\prime}=2 \pi l_{1}^{2} i \frac{1}{\gamma} K_{1}\left(\frac{k R}{\gamma m}\right) \boldsymbol{\mu},
$$

where $\boldsymbol{\mu}=\boldsymbol{R} / R$ and $m=l_{2} / l_{1}$ is the magnification factor of the optical system.

In the second integral we assume $\theta_{a} \gg \gamma^{-1}$ and neglect $\gamma^{-2}$ in the denominator to obtain

$$
l_{1} \int_{\text {out }} e^{-i k \boldsymbol{R} \cdot \boldsymbol{r}^{\prime} / l_{2}} \frac{\boldsymbol{r}^{\prime}}{r^{\prime 2}} d^{2} r^{\prime}
$$

Due to symmetry of the integand this integral is directed along $\boldsymbol{R}$ and can be written as $B \boldsymbol{\mu}$ with

$$
\begin{aligned}
B & =l_{1} \int_{\mathrm{c}} e^{-i k \boldsymbol{R} \cdot \boldsymbol{r}^{\prime} / l_{2}} \frac{\boldsymbol{\mu} \cdot \boldsymbol{r}^{\prime}}{r^{\prime 2}} d^{2} r^{\prime}=l_{1} \int_{a}^{\infty} d r^{\prime} \int_{0}^{2 \pi} d \phi e^{-i k R r^{\prime} \cos \phi / l_{2}} \cos \phi \\
& =-2 i \pi l_{1} \int_{a}^{\infty} d r^{\prime} J_{1}\left(\frac{k R r^{\prime}}{l_{2}}\right)=-2 i \pi l_{1} \frac{l_{2}}{k R} J_{0}\left(\frac{k R a}{l_{2}}\right)
\end{aligned}
$$

where we accomplished the integration in the polar coordinate system with the angle $\phi$ between the vectors $\boldsymbol{\mu}$ and $\boldsymbol{r}^{\prime}$.

Combining expressions (A3)-(A5) we find

$$
I_{\text {lens }}=2 \pi i \boldsymbol{\mu} l_{1}^{2}\left[\frac{1}{\gamma} K_{1}\left(\frac{k R}{\gamma m}\right)-\frac{m}{k R} J_{0}\left(\frac{k R \theta_{a}}{m}\right)\right] .
$$

Since we are interested in the distribution of the electric field in the focus, which typically extends over the region $k R \sim 1$, in the limit $\gamma \gg 1$ we can use the asymptotic expression $K_{1}(k R / \gamma m) \approx \gamma m / k R$ which gives

$$
I_{\mathrm{lens}}=2 \pi i \boldsymbol{\mu} l_{1}^{2} \frac{m}{k R}\left[1-J_{0}\left(\frac{k R \theta_{a}}{m}\right)\right] .
$$


[1] H. Loos, T. Borden, P. Emma, J. Frisch, and J. Wu, in Proceedings of the 2007 Particle Accelerator Conference (IEEE, 2007) p. 4189.

[2] T. Maxwell, private communication.

[3] V. A. Lebedev, Nucl. Inst. and Meth. A 372, 344 (1996).

[4] H. Loos, R. Akre, A. Brachmann, F.-J. Decker, Y. Ding, D. Dowell, P. Emma, J. Frisch, S. Gilevich, G. Hays, P. Hering, Z. Huang, R. Iverson, C. LimborgDeprey, A. Miahnahri, S. Molloy, H.-D. Nuhn, J. Turner, J. Welch, W. White, J. Wu, and D. Ratner, in Proceedings of the 2008 FEL Conference, paper THBAU01 (Gyeongju, Korea, 2008).

[5] J. D. Jackson, Classical Electrodynamics, 3rd ed. (Wiley, New York, 1999).

[6] M. L. Ter-Mikaelian, High-Energy Electromagnetic Processes in Condensed Media (Wiley-Interscience, New York, 1972).

[7] B. M. Bolotovskii and E. A. Galst'yan, Physics-Uspekhi 43, 755 (2000).

[8] K. K. Sharma, Optics principles and applications (Academic Press, 2006). 